\title{
Renal Dynamic Imaging Information Data in the Evaluation of Renal Function in Patients with Chronic Heart Failure under the Guidance of Intelligent Region of Interest Detection Algorithm
}

\author{
Haifang Ma $\mathbb{D}^{1},{ }^{1}$ Zhenjun Su $\mathbb{D}^{2},{ }^{2}$ Zhijia Yang $\mathbb{D}^{3},{ }^{3}$ Ya Li $\mathbb{D},{ }^{1}$ Xiaoli Yang $\mathbb{D}^{4},{ }^{4}$ Danhua Zhao $\mathbb{D}^{1}$, \\ and Xuejiao Chen $\mathbb{1 0}^{5}$ \\ ${ }^{1}$ Department of Cardiovascular Medicine, Affiliated Hospital of Hebei University of Engineering, Handan 056002, Hebei, China \\ ${ }^{2}$ Department of Nuclear Medicine, Affiliated Hospital of Hebei University of Engineering, Handan 056002, Hebei, China \\ ${ }^{3}$ Department of Cardiovascular Medicine, Handan Central Hospital, Handan 056001, Hebei, China \\ ${ }^{4}$ Department of Neurology, Affiliated Hospital of Hebei University of Engineering, Handan 056002, Hebei, China \\ ${ }^{5}$ Department of Medical Administration, Affiliated Hospital of Hebei University of Engineering, Handan 056002, Hebei, China
}

Correspondence should be addressed to Xuejiao Chen; liwei@hebeu.edu.cn

Received 25 October 2021; Revised 13 January 2022; Accepted 18 January 2022; Published 28 February 2022

Academic Editor: M Pallikonda Rajasekaran

Copyright (c) 2022 Haifang Ma et al. This is an open access article distributed under the Creative Commons Attribution License, which permits unrestricted use, distribution, and reproduction in any medium, provided the original work is properly cited.

This work aimed to explore the application value of intelligent region of interest (ROI) detection algorithm in evaluating renal dynamic imaging information data of renal function in patients with chronic heart failure (CHF). 52 patients with CHF were selected as the research objects and grouped according to the level of renal function into an experimental group (14 cases) and an observation group (38 cases). In addition, 10 healthy people were selected as the research objects, as the control group. Different calculation methods of glomerular filtration rate (GFR) were used to evaluate the renal function of patients, and the ROI detection algorithm-assisted delineation was compared with traditional manual delineation. It was found that the GFR of the experimental group calculated by different methods was $52.58 \mathrm{~mL} / \mathrm{min} / 1.73 \mathrm{~m} 2$ and $43.77 \mathrm{~mL} / \mathrm{min} / 1.73 \mathrm{~m} 2$, respectively, which was significantly lower than that of the observation group $(P<0.05)$, and that in both groups was lower than that of the control group $(P<0.05)$. In addition, the unilateral renal function values of the two auxiliary delineations were both $13.61 \mathrm{~mL} / \mathrm{min} / 1.73 \mathrm{~m}^{2}$, while the results of the two manual delineations were significantly different $(P<0.05)$. Therefore, renal dynamic imaging based on the ROI detection algorithm to evaluate the renal function of CHF patients showed a great improvement in effectiveness, sensitivity, stability, and efficiency compared with the traditional manual delineation, which was worthy of clinical promotion.

\section{Introduction}

Heart failure refers to the dysfunction of the systolic and diastolic functions of the heart caused by various cardiovascular diseases. It is unable to completely exclude the heart from the venous return blood, resulting in venous blood stasis and insufficient arterial blood supply, causing various manifestations of organ ischemia [1-3].

Almost all cardiovascular diseases can induce heart failure $[4,5]$, and some common factors can also lead to heart failure, including respiratory tract infection, severe arrhythmia, excessive cardiac load, and excessive emotions, which will all change the structure and function of the myocardium, resulting in insufficient ventricular pumping function and filling capacity [6, 7]. Heart failure can cause dysfunction of various organs such as the kidneys, lungs, and liver, while abnormal organ function will further aggravate heart failure [8-10]. Therefore, the evaluation of various organ functions is an important indicator of the prognosis of heart failure [11].

The kidney is one of the vital organs of the human body. Its basic function is to remove metabolites and some harmful substances from the body by using glomerular filtration and reabsorption of renal tubules, while retaining water and 
other useful substances, so as to balance water and electrolytes in the body to maintain acid-base balance [12]. In the working mechanism of kidney, the filtration function of glomerular plays the most important role. There are about one million glomeruli in the kidney of each person, and blood filters through the capillary basement membrane of the semipermeable glomerulus to produce plasma ultrafiltrate (i.e., protopuria). This process is called glomerular filtration. Glomerular filtration rate (GFR) is an important and sensitive evaluation data in renal function evaluation. Renal dynamic imaging is commonly used to measure the glomerular filtration level $[13,14]$.

The region of interest (ROI) is a key mark in the image to assist doctors in diagnosing the relevant parts after the lesion and the contrast agent in the dynamic imaging of the kidney reach a certain concentration. However, due to the existence of technical problems, the current area of interest is mostly delineated manually by relevant personnel on the software. On this basis, a targeted intelligent ROI detection algorithm was combined to replace the traditional renal dynamic imaging method of manually delineating the ROI by doctors, which can effectively improve the accuracy of the captured images and the accuracy of ROI delineation [15-17]. To sum up, the intelligent ROI detection algorithm was combined with renal dynamic imaging to evaluate renal function according to the information data, so as to improve the clinical evaluation ability of renal function in CHF patients and better assess the patient's prognosis.

\section{Materials and Methods}

2.1. Research Objects. Fifty-two patients with CHF were selected, who were diagnosed with grade II heart failure. A total of 14 patients with split renal function (SRF) $<30 \mathrm{~mL} / \mathrm{min} /$ $1.73 \mathrm{~m}^{2}$ were selected as the experimental group; a total of 38 cases with $30 \mathrm{~mL} / \mathrm{min} / 1.73 \mathrm{~m}^{2}<\mathrm{SRF}<50 \mathrm{~mL} / \mathrm{min} / 1.73 \mathrm{~m}^{2}$ were selected as the observation group. All subjects included 24 males and 18 females, aged 47-63 years old, with an average age of $54.23 \pm 7.59$ years. The main clinical symptoms of the patients were 21 cases of hypertension, 13 cases of coronary heart disease, 11 cases of valvular disease, and 7 cases of cardiomyopathy. The age, gender, and type of heart disease in the two groups were not statistically significant. In addition, 10 healthy people were selected from the hospital database as the control group, including 5 males and 5 females, aged from 35 to 54 years, with an average age of $44 \pm 10.82$ years. This study had been approved by the Ethics Committee of the hospital, and the patients and their families had understood the situation of the study and signed the informed consent forms.

The inclusion criteria were defined as follows: patients who were diagnosed with grade II heart failure according to evaluation criteria given by the New York Heart Association (NYHA) [18] and patients with no history of taking diuretic drugs three days before the experiment.

The exclusion criteria were defined as follows: patients with a history of kidney disease, diabetes, and tumor; patients with severe mental illness and unable to communicate normally; and patients whose families and patients were unwilling to participate.

2.2. Research Plan and Process. All cases that met the access requirement were scanned with the intelligent ROI detection algorithm-based renal dynamic imaging. The instrument was Infinia dual-probe single-photon emission computed tomography (SPECT), with a low-energy high-resolution parallel-hole collimator. Before the examination, the patient was required to have an empty stomach and drink $300 \mathrm{~mL}$ of water half an hour in advance. During the examination, he was lying face up in bed and injected with 99Tcm-DTPA (99m-technetium-diethyltriaminepentaacetic acid) into the elbow vein. The energy peak was $140 \mathrm{keV}$, the window width was $\pm 20 \%$, and the plane acquisition matrix was $64 \times 64$. At the same time, when the tracer was injected for 2 hours (T1) and 4 hours (T2), $4 \mathrm{~mL}$ of blood was taken from the cubital vein, anticoagulated with heparin, and centrifuged at $38870 \mathrm{rpm}$ for 10 minutes. Plasma was detected by radioimmunoassay $\gamma$-counter. According to the collected test data, the two groups of rGFR (equation (1)) and gGFR (equation (2)) were calculated, and the rGFR and gGFR were compared and undertaken as the evaluation criteria [19].

$$
\begin{aligned}
\mathrm{rGFR} & =\frac{\mathrm{CIn} /(P 1 / P 2) / T 2-T 1 \exp [T 1 \operatorname{In} P 2-(T 2 \operatorname{In} P 1)] / T 2-T 1}{\mathrm{BSA} / 1.73}, \\
\mathrm{gGFR} & =\left(\frac{P_{R K}-P_{R B} / e^{-\mu D_{R}}+P_{L K}-P_{L B} / e^{-\mu D_{L}}}{P_{\text {pre }}-P_{\text {post }}} \times 100\right) \times 9.813-6.825, \\
\mathrm{BSA} & =0.007184 \times H^{0.725} \times W^{0.425}, \\
D_{R} & =13.3 \times \frac{W}{H}+0.7, \\
D_{L} & =13.2 \times \frac{W}{H}+0.7,
\end{aligned}
$$


where $C$ was the radioactivity (cpm); $P 1$ and $P 2$ referred to the radioactivity count at time $T 1$ and time $T 2$, respectively; $P_{R K}$ and $P_{R B}$ represented the count of right kidney radioactivity and the right kidney background radioactivity, respectively; $P_{L K}$ and $P_{L B}$ referred to the count of the left kidney radioactivity and left kidney background radioactivity, respectively; $P_{\text {pre }}$ and $P_{\text {post }}$ referred to the tracer radioactivity count before syringe injection and the count of residual tumor radioactivity of the tracer after syringe injection, respectively; $D_{R}$ was the distance between the right kidney and the body surface; $D_{L}$ was the distance between the left kidney and the body surface; $H$ was the height $(\mathrm{cm})$, $W$ was the weight $(\mathrm{kg})$; and $\mu$ referred to the correction coefficient with the size of -0.153 .

\subsection{Dynamic Renal Imaging Based on Intelligent ROI De-} tection Algorithm. Regarding the ROI, traditional renal dynamic imaging is manually outlined by relevant professionals. From the above equation, it can be clearly found that the size of GFR was closely related to the delineation of the ROI in the kidney and the background ROI, while the accuracy and speed of the manual delineation ROI were not satisfactory. Therefore, an intelligent ROI segmentation method based on single-photon emission computed tomography (SPECT) was proposed in this study, which could eliminate the error caused by the manual delineation ROI to the greatest extent and increase the selection rate while ensuring the accuracy [20].

Since the ROI was selected on the original synthetic image of the kidney, it had to preprocess the synthetic image firstly in this study. Because of the influence on the ROI of the image, the extrarenal background brightness area had to be removed firstly, and the removal result is shown in Figure 1. At the same time, image enhancement was performed on the remaining areas to improve image recognition.

Image enhancement could select an intensity-pair enhancement algorithm for image processing. The algorithm calculated the intensity difference based on the intensity-pair selection rule for each pixel in the image, established an expansion or de-expansion function based on the intensity difference, and calculated an intensity mapping function to suppress image noise and enhance image edge contrast. The specific algorithm was to firstly normalize the image pixel value to the interval $[0,255]$, and $A(x, y)$ was assumed as the pixel intensity of the image at the point $(x, y)$ in the image. All pixels were scanned, and the selected pixel intensity $A$ ( $x$, $y$ ) and the intensity difference of the nearby four pixel intensities $A(x-1, y), A(x, y-1), A(x-1, y-1)$, and $\mathrm{A}(x+1$, $y-1)$ were calculated. After the intensity difference was obtained, the image can be enhanced according to the process shown in Figure 2.

After the enhanced image was obtained, the ROI was acquired. In this study, an image segmentation technique based on image edge segmentation was adopted to grid the kidney area. Similar to the image enhancement method, point $B(x, y)$ was also selected, and the difference value was calculated with 4 adjacent area pixels around this point. When the difference exceeded a certain threshold, the pixel can be considered to be in the edge area. After calculation of all the pixels of the image, the kidney edge image $C(x, y)$ can be calculated:

$C(x, y)=\left\{\begin{array}{l}255, \text { if } \operatorname{abs}(B(x, y)-B(x, y-1)) \geq 0.044 \times B_{\max }, \\ \operatorname{abs}(B(x, y)-B(x-1, y)) \geq 0.044 \times B_{\max }, \\ 0, \text { others, }\end{array}\right.$

where $B_{\max }$ was the maximum value of the pixels in the kidney area of the preliminary ROI.

After the ROI in the kidney was obtained, the background ROI can be obtained through automatic image processing.

2.4. Simulation Experiment and Its Evaluation Indicators. To simulate the effect of image enhancement, the most intuitive method usually used is to calculate the mean square error (MSE) and peak signal-to-noise ratio (PSNR) of the enhanced image and the original image. The MSE could be calculated with the following equation:

$$
\text { mean square error }=\frac{1}{L \times W} \sum_{i=1}^{L} \sum_{j=1}^{W}\left(C_{1}(i, j)-C_{2}(i, j)\right)^{2} \text {, }
$$

where $C_{1}$ was the enhanced image, $C_{2}$ was the original image, $L$ referred to the image length $(\mathrm{cm})$, and $W$ referred to the image width $(\mathrm{cm})$; the smaller the MSE, the finer the reconstructed image.

The PSNR was the most commonly used evaluation index in the field of image processing and could be calculated with the following equation:

$$
\text { peak signal }- \text { to }- \text { noise ratio }=10 \log _{10}\left(\frac{\left(2^{b}-1\right)^{2}}{\mathrm{MSE}}\right),
$$

where $b$ represented the number of bits per pixel, and the larger the PSNR, the higher the quality of image reconstruction.

In this study, MSE and PSNR of the images before and after enhancement were calculated and compared to verify the effectiveness of image enhancement.

The experimental environment was set as follows: Win 10 system, central processing unit (CPU) - Intel(R) Celeron(R) CPU1007U@1.50 GHz, and MATLAB platform.

2.5. Statistical Analysis. In this study, data were processed using SPSS (Statistical Product and Service Solutions) to calculate gGFR and rGFR. One-way analysis of variance was used for pairwise comparison. The difference was statistically significant at $P<0.05$.

\section{Results}

3.1. Simulation Experiment Results of the Algorithm. Figure 3 shows the original images of 4 time periods in the renal dynamic imaging of a 48-year-old female patient.

Images in Figure 3 were enhanced according to the process of Figure 2, and the output is shown in Figure 4. 


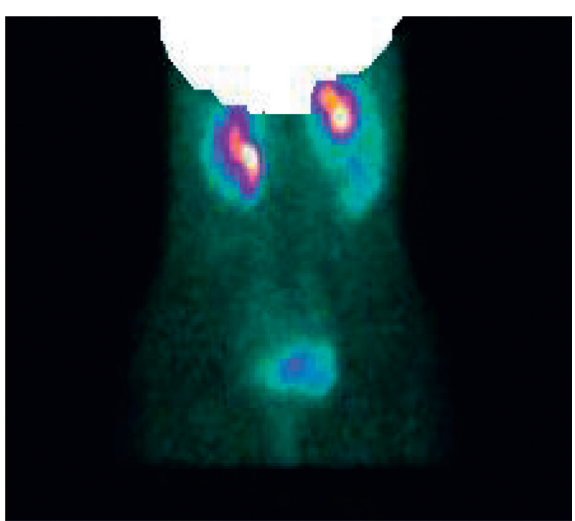

(a)

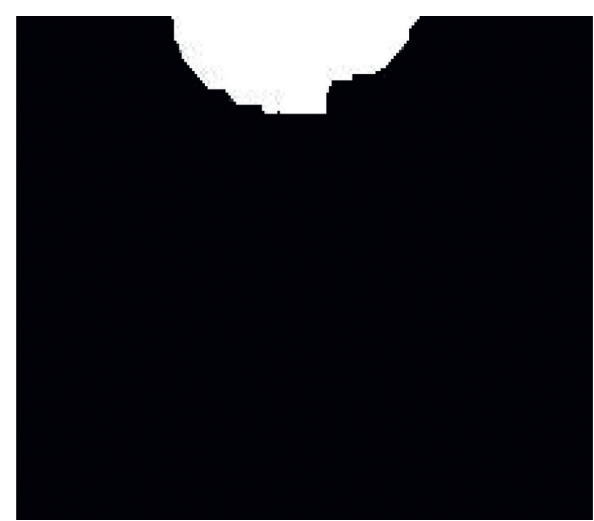

(b)

Figure 1: The result of extrarenal background removal. (a) The result after background removal. (b) The extracted space segmentation boundary template.

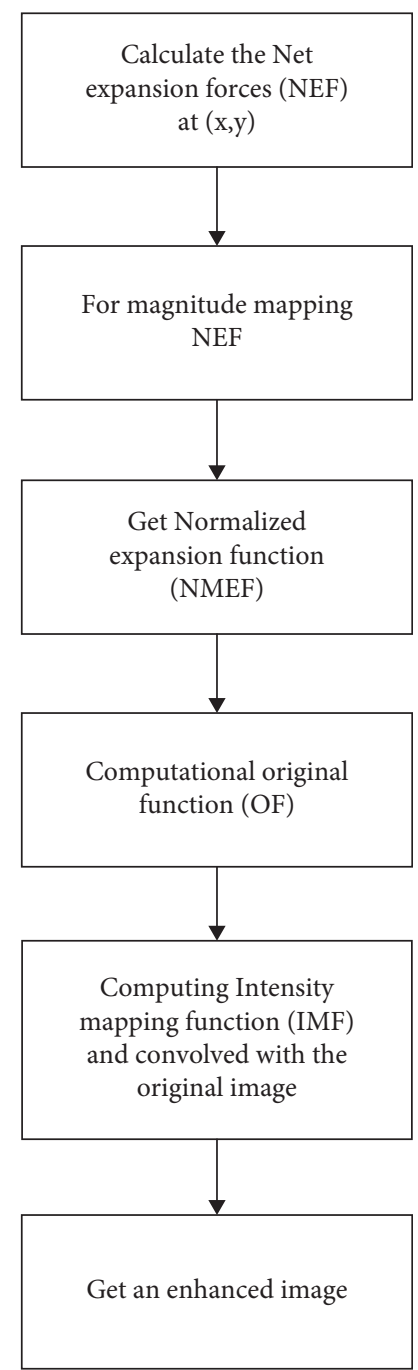

FIGURE 2: The specific flowchart of image enhancement. 


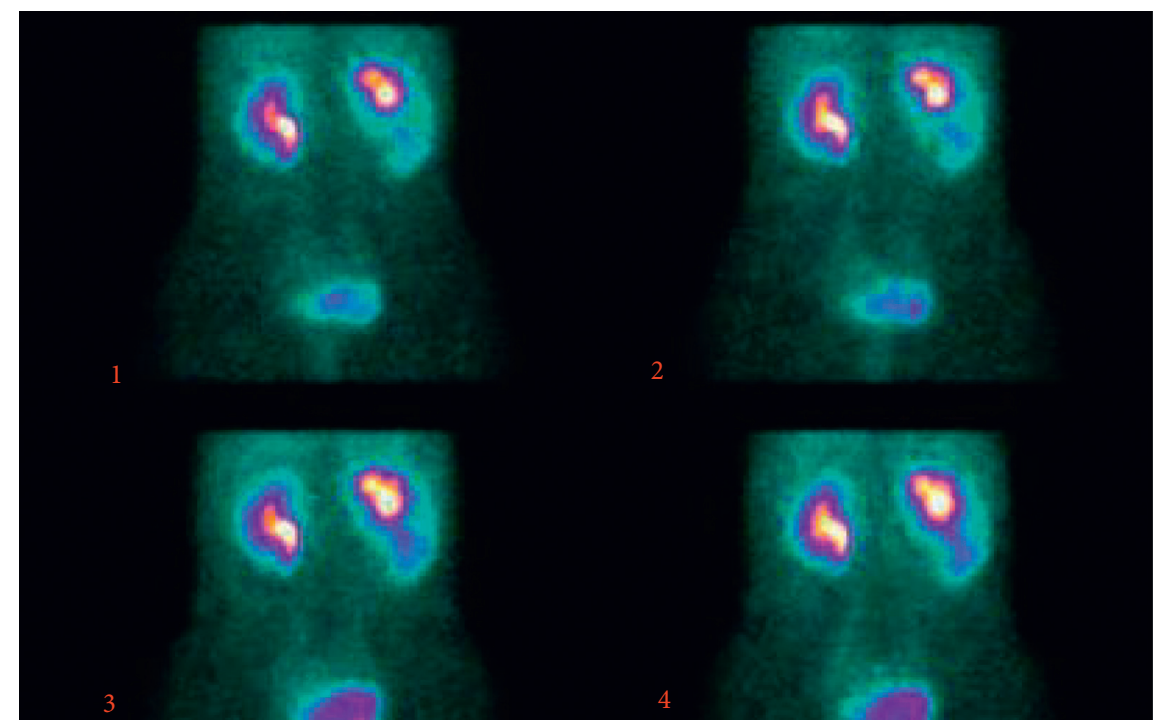

FIGURE 3: Screenshot of the patient's original renal dynamic imaging. (a), (b), (c), and (d) represented different time periods.

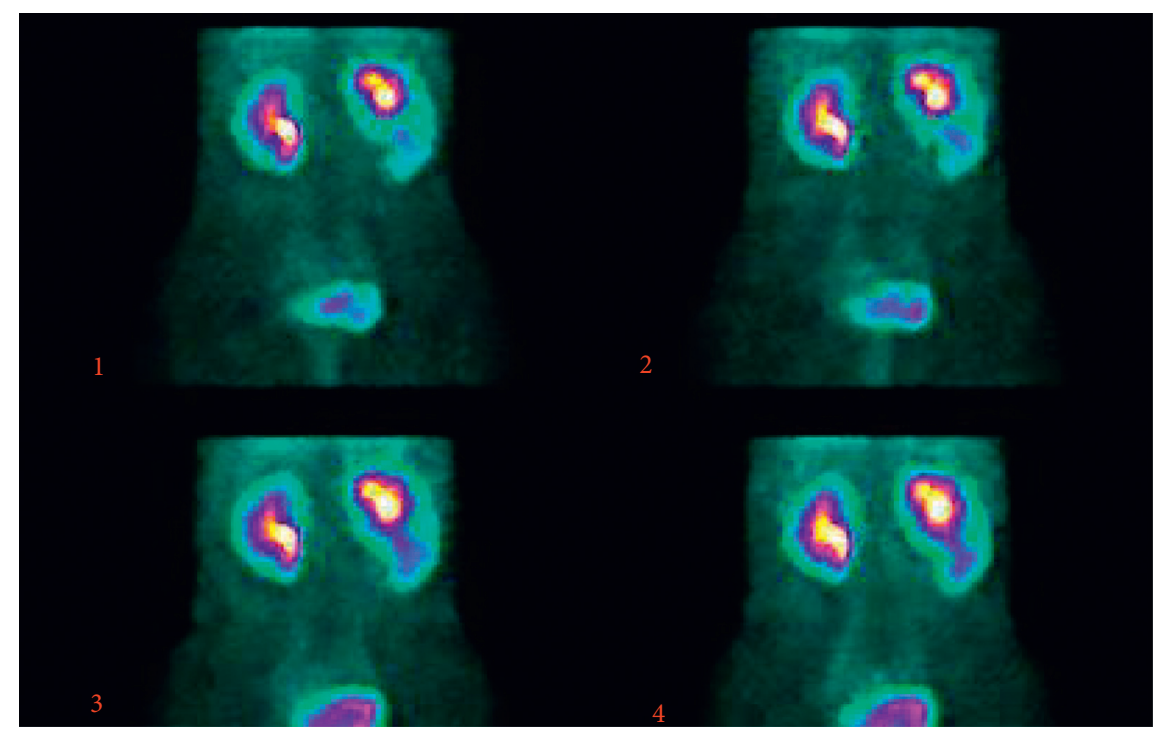

FIGURE 4: Image enhancement result. (a), (b), (c), and (d) represented different time periods.

The corresponding PSNR and MSE comparison is shown in Figure 5.

As shown in Figure 5, after the image was enhanced by the intelligent ROI detection algorithm, the mean square error (MSE) dropped to $0.0032 \mathrm{~cm}$, and the peak signal-tonoise ratio (PSNR) increased to $21.94 \mathrm{~dB}$. It meant that the image of the kidney area was obviously enhanced after processing.

3.2. The Performance of the ROI in the Kidney. The kidney ROI and background ROI were delineated on the enhanced image, and the delineation result is shown in Figure 6.

3.3. Evaluation of Renal Function. Figure 7 shows the comparison of the calculation results of gGFR and rGFR for all experimental subjects in different groups.
As demonstrated in Figure 7, the gGFR and rGFR of the experimental group were $52.58 \mathrm{~mL} / \mathrm{min} / 1.73 \mathrm{~m}^{2}$ and $43.77 \mathrm{~mL} / \mathrm{min} / 1.73 \mathrm{~m}^{2}$, respectively; the gGFR and rGFR of the observation group were $79.31 \mathrm{~mL} / \mathrm{min} / 1.73 \mathrm{~m}^{2}$ and $68.47 \mathrm{~mL} / \mathrm{min} / 1.73 \mathrm{~m}^{2}$, respectively; and those in the control group were $94.26 \mathrm{~mL} / \mathrm{min} / 1.73 \mathrm{~m}^{2}$ and $98.91 \mathrm{~mL} / \mathrm{min} /$ $1.73 \mathrm{~m}^{2}$, respectively. It suggested that the values of the experimental group were lower than those of the observation group, and the values in both the experimental group and the observation group were lower than those of the control group $(P<0.05)$.

In this study, the intelligent ROI detection algorithm and manual delineation proposed in this study were adopted to delineate the same kidney dynamic imaging. Two sketching methods were used to sketch twice, and the SRF was calculated according to the sketching results for comparison, as shown in Figure 8. 


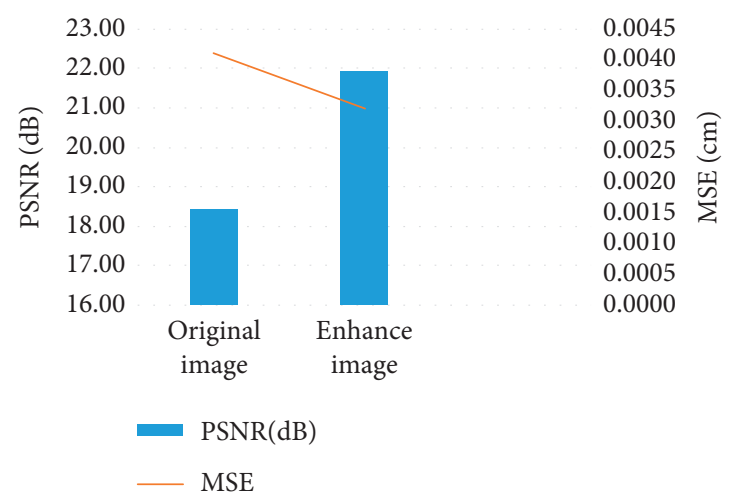

FIGURE 5: Comparison of enhanced image enhancement effects.

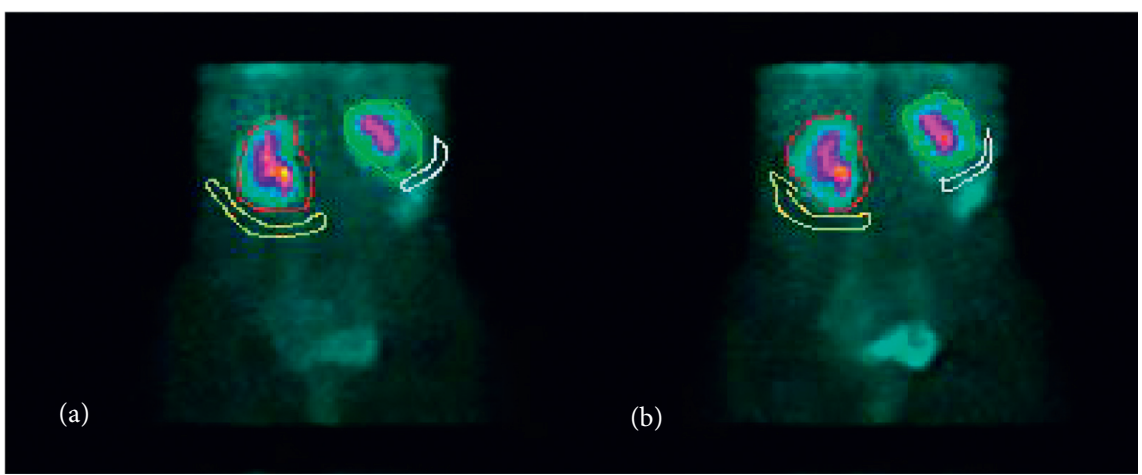

FIgURE 6: Dynamic imaging of renal region of interest. (a) The intelligent delineation results of a frame of renal region of interest. (b) The result of a frame of renal region of interest manually sketched.

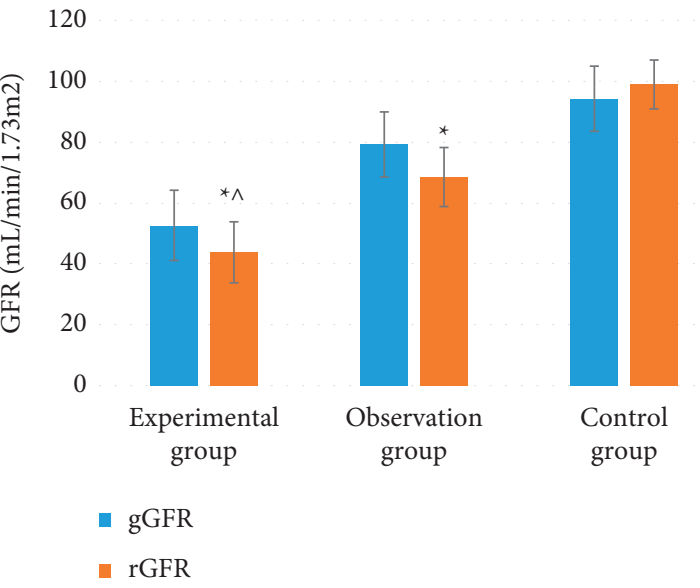

FIGURE 7: Comparison of gGFR and rGFR between the experimental group and the control group (* and ^ meant that compared with the control group, the difference in the experimental group showed $P<0.05$ and the difference in the observation group showed $P<0.05$, respectively).

Figure 8 reveals that the unilateral renal function values of the first and second manual delineation were $15.48 \mathrm{~mL} /$ $\mathrm{min} / 1.73 \mathrm{~m}^{2}$ and $19.25 \mathrm{~mL} / \mathrm{min} / 1.73 \mathrm{~m}^{2}$, respectively, and the unilateral renal function values of the first and second auxiliary delineation were both $13.61 \mathrm{~mL} / \mathrm{min} / 1.73 \mathrm{~m}^{2}$. There were obvious deviations between the two results of

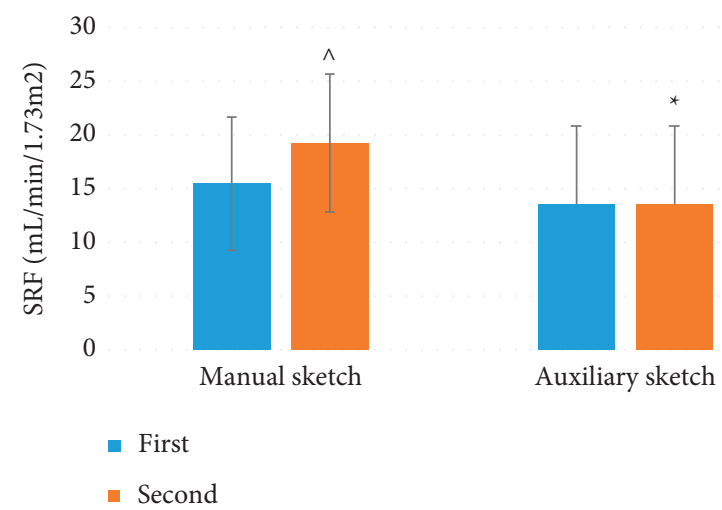

FIGURE 8: Comparison of unilateral renal function values between manual delineation and auxiliary delineation $\left({ }^{*}\right.$ and ${ }^{\wedge}$ meant that the difference of auxiliary delineation and manual delineation showed $P>0.05$ and $P<0.05$ compared with the first delineation, respectively).

manual delineation, and the difference was statistically significant $(P<0.05)$; there was no significant difference in the results of auxiliary delineation $(P>0.05)$.

\section{Discussion}

Relevant literature records that renal dynamic imaging, as the preferred method for detecting kidney morphology 
and function, plays an important role in many aspects such as renal blood supply, renal function, and renal urinary tract [21]. By selecting the region of interest in renal dynamic imaging, the high concentration area of imaging agent in the diseased organs can be selected, which greatly facilitates the diagnosis of the patient's examination site and saves a lot of meaningless and repetitive work. However, in renal dynamic imaging, the image will be interfered by many factors and the technology is not perfect. At present, it is mostly done manually by professionals, which is not only inefficient but also affected by the subjective consciousness of the operator [22,23]. Therefore, the artificial intelligence was combined with traditional manual work in this work, and the intelligent algorithms were adopted to select the contours of the ROI, which was then manually calibrated by professionals. Such way not only improves the accuracy of the selection but also reduces the operation time, showing the combination of advantages of two methods.

Pang et al. [24] proposed a new calculation equation nGFR to verify the effectiveness of GFR in renal function verification and compared the new equations with gGFR, tGFR, cGFR, and aGFR. The results showed that the new equations showed better repeatability and effectiveness than other equations. The results showed that the new equation had better repeatability and validity than other formulas. This is exactly the same as the method in this work. The initial image of renal dynamic imaging is relatively blurry, and the region of interest will be affected by other imaging parts. Therefore, it took the lead in enhancing the image to improve the clarity while eliminating the interference in this work. After that, simulation experiments were carried out on the image after enhancement and before enhancement. It was found that after the image was enhanced by the intelligent ROI detection algorithm, the MSE decreased to $0.0032 \mathrm{~cm}$, and the PSNR increased to $21.94 \mathrm{~dB}$. It can be concluded that the image enhancement algorithm is very effective.

The gGFR and rGFR of the experimental group were $52.58 \mathrm{~mL} / \mathrm{min} / 1.73 \mathrm{~m}^{2}$ and $43.77 \mathrm{~mL} / \mathrm{min} / 1.73 \mathrm{~m}^{2}$, respectively; those of the observation group were $79.31 \mathrm{~mL} /$ $\mathrm{min} / 1.73 \mathrm{~m}^{2}$ and $68.47 \mathrm{~mL} / \mathrm{min} / 1.73 \mathrm{~m}^{2}$, respectively; and those of the control group were $94.26 \mathrm{~mL} / \mathrm{min} /$ $1.73 \mathrm{~m}^{2}$ and $98.91 \mathrm{~mL} / \mathrm{min} / 1.73 \mathrm{~m}^{2}$, respectively. It revealed that the values of the experimental group were lower than those of the observation group, and values in both the experimental group and the observation group were lower than those of the control group $(P<0.05)$. It shows that the renal function of patients with CHF will be significantly lower than that of healthy people, and the detection method can effectively distinguish different degrees of renal function damage and is clearly reflected in the experimental data. Ma et al. [25] calculated the information of renal dynamic imaging data by using three different calculation methods for 482 patients with chronic kidney disease. The results showed that the MDRD equation based on the modified disease diet adjustment research equation had no significant difference with GFR in the evaluation of renal function, and
GFR still showed reference value especially in a large number of samples. In this work, the same kidney dynamic image was delineated twice using the intelligent region of interest detection algorithm and manual delineation. It was found that the unilateral renal function values of the first and second manual delineation were $15.48 \mathrm{~mL} / \mathrm{min} / 1.73 \mathrm{~m}^{2}$ and $19.25 \mathrm{~mL} / \mathrm{min} / 1.73 \mathrm{~m}^{2}$, respectively; the unilateral renal function values of the first and second auxiliary delineation were both $13.61 \mathrm{~mL} /$ $\min / 1.73 \mathrm{~m}^{2}$. There was a significant deviation between the two results of manual delineation $(P<0.05)$, but there was no significant difference in the results of the auxiliary delineation proposed $(P>0.05)$. It can be concluded that the manual delineation method has obvious individual differences, while the auxiliary delineation results are very stable, and the fluctuation between the results is small. Such results prove that the stability of the auxiliary delineation has been significantly strengthened after repeated experiments.

In conclusion, it was determined that the renal dynamic imaging based on the intelligent ROI detection algorithm was more effective, sensitive, stable, and useful than the traditional manual delineation in the evaluation of renal function in patients with CHF. The efficiency had been significantly improved, and it can be promoted and used clinically as an upgraded version of the traditional renal function evaluation method for manually delineating ROI.

\section{Conclusion}

In this study, $52 \mathrm{CHF}$ patients were selected as the research objects, and the renal dynamic imaging based on the intelligent ROI detection algorithm was performed, and the results of the proposed algorithm were compared with those of traditional manual delineation. The results proved that the renal function of CHF patients evaluated by the intelligent ROI detection algorithm based on the dynamic renal function was greatly improved in terms of effectiveness, sensitivity, stability, and efficiency compared with the traditional manual delineation, so it was worthy of clinical promotion. However, a more convenient pixel boundary segmentation algorithm was adopted in this study, and there may still be room for improvement in image processing efficiency. With the iteration of the algorithm in the future, choosing a smarter algorithm for image segmentation may further improve the results. This study could provide a more effective, stable, and convenient evaluation method for clinical renal function evaluation through the auxiliary application of intelligent algorithms.

\section{Data Availability}

The data used to support the findings of this study are available from the corresponding author upon request.

\section{Conflicts of Interest}

The authors declare that they have no conflicts of interest. 


\section{References}

[1] K. F. Adams, G. C. Fonarow, C. L. Emerman et al., "Characteristics and outcomes of patients hospitalized for heart failure in the United States: rationale, design, and preliminary observations from the first 100,000 cases in the Acute Decompensated Heart Failure National Registry (ADHERE)," American Heart Journal, vol. 149, no. 2, pp. 209-216, 2005.

[2] C. Wittenbecher, F. Eichelmann, E. Toledo et al., "Lipid profiles and heart failure risk," Circulation Research, vol. 128, no. 3, pp. 309-320, 2021.

[3] W. T. Abraham, J. Lindenfeld, P. Ponikowski et al., "Effect of empagliflozin on exercise ability and symptoms in heart failure patients with reduced and preserved ejection fraction, with and without type 2 diabetes," European Heart Journal, vol. 42, no. 6, pp. 700-710, 2021.

[4] M. Hu, Y. Zhong, S. Xie, H. Lv, and Z. Lv, "Fuzzy system based medical image processing for brain disease prediction," Frontiers in Neuroscience, vol. 15, Article ID 714318, 2021.

[5] D. Kotecha, C. S. P. Lam, D. J. Van Veldhuisen, I. C. Van Gelder, A. A. Voors, and M. Rienstra, "Heart failure with preserved ejection fraction and atrial fibrillation," Journal of the American College of Cardiology, vol. 68, no. 20, pp. 2217-2228, 2016.

[6] B. Pieske, C. Tschöpe, R. A. de Boer et al., "How to diagnose heart failure with preserved ejection fraction: the HFA-PEFF diagnostic algorithm: a consensus recommendation from the Heart Failure Association (HFA) of the European Society of Cardiology (ESC)," European Heart Journal, vol. 40, no. 40, pp. 3297-3317, 2019.

[7] N. Nair, "Epidemiology and pathogenesis of heart failure with preserved ejection fraction," Reviews in Cardiovascular Medicine, vol. 21, no. 4, pp. 531-540, 2020 Dec 30.

[8] Z. Lv and L. Qiao, "Analysis of healthcare big data," Future Generation Computer Systems, vol. 109, pp. 103-110, 2020.

[9] S. Gottlieb, "Drug cuts deaths from heart failure by a third," $B M J$, vol. 319 , no. 7205 , p. 276,1999

[10] K. E. Di Palo and N. J. Barone, "Hypertension and heart failure," Heart Failure Clinics, vol. 16, no. 1, pp. 99-106, 2020.

[11] M. Gowrishankar, C. VanderPluym, C. Robert, F. Bamforth, S. Gilmour, and A. Senthilselvan, "Value of serum cystatin C in estimating renal function in children with non-renal solid organ transplantation," Pediatric Transplantation, vol. 19, no. 1, pp. 27-34, 2015.

[12] C. P. Liu, Y. Hu, J. C. Lin, H. L. Fu, L. Y. Lim, and Z. X. Yuan, "Targeting strategies for drug delivery to the kidney: from renal glomeruli to tubules," Medicinal Research Reviews, vol. 39, no. 2, pp. 561-578, 2019.

[13] M. Hornum and B. Feldt-Rasmussen, "Glomerular filtration rate estimation in renal and non-renal solid organ transplantation," Nephron, vol. 136, no. 4, pp. 298-301, 2017.

[14] M. Song, Y. Yue, Y. Jin et al., "Intravoxel incoherent motion and ADC measurements for differentiating benign from malignant thyroid nodules: utilizing the most repeatable region of interest delineation at 3.0 T," Cancer Imaging, vol. 20, no. 1 , p. 9, 2020.

[15] T. Calderbank, M. Bown, and A. Saratzis, "The impact of suprarenal fixation on renal function following endovascular abdominal aortic aneurysm repair: meta-analysis based on estimated glomerular filtration rate," European Journal of Vascular and Endovascular Surgery, vol. 56, no. 4, pp. 497506, 2018.

[16] D. A. Baran, C. L. Grines, S. Bailey et al., "SCAI clinical expert consensus statement on the classification of cardiogenic shock," Catheterization and Cardiovascular Interventions, vol. 94, no. 1, pp. 29-37, 2019.

[17] H. Zhang, Y. Li, Z. Lv, A. K. Sangaiah, and T. Huang, "A realtime and ubiquitous network attack detection based on deep belief network and support vector machine," IEEE/CAA Journal of Automatica Sinica, vol. 7, no. 3, pp. 790-799, 2020.

[18] C. Caraballo, N. R. Desai, H. Mulder et al., "Clinical implications of the New York heart association classification," Journal of American Heart Association, vol. 8, no. 23, Article ID e014240, 2019 Dec 3.

[19] Q. Li, C.-l. Zhang, Z.-1. Fu, R.-f. Wang, Y.-c. Ma, and L. Zuo, "Development of formulae for accurate measurement of the glomerular filtration rate by renal dynamic imaging," Nuclear Medicine Communications, vol. 28, no. 5, pp. 407-413, 2007.

[20] X. Jing, X. Zheng, S. Song, and K. Liu, "[A computer tomography assisted method for the automatic detection of region of interest in dynamic kidney images]," Sheng $W u Y i$ Xue Gong Cheng Xue Za Zhi, vol. 34, no. 6, pp. 928-933, 2017, Chinese.

[21] C. Wang, C. Gao, W. Maimaiti, S. Li, Q. Yang, and L. Jiang, "The features of technetium-99m-DTPA renal dynamic imaging after severe unilateral ureteral obstruction in adult rabbits," PLoS One, vol. 15, no. 8, Article ID e0237443, 2020.

[22] A. Lubas, G. Kade, R. Ryczek et al., "Ultrasonic evaluation of renal cortex arterial area enables differentiation between hypertensive and glomerulonephritis-related chronic kidney disease," International Urology and Nephrology, vol. 49, no. 9, pp. 1627-1635, 2017.

[23] L. Wang, K. Chen, and Q. Xu, "The application of $99 \mathrm{mTc}$ DTPA renal dynamic imaging to measuring renal function of children with acute lymphoblastic leukemia after induction therapy," BioMed Research International, vol. 2020, Article ID 3687134, 2020.

[24] X. Pang, F. Li, S. Huang et al., "A novel method for accurate quantification of split glomerular filtration rate using combination of Tc-99m-DTPA renal dynamic imaging and its plasma clearance," Disease Markers, vol. 2021, Article ID 6643586, 2021.

[25] Y.-C. Ma, L. Zuo, C.-L. Zhang, M. Wang, R.-F. Wang, and H.-Y. Wang, "Comparison of 99mTc-DTPA renal dynamic imaging with modified MDRD equation for glomerular filtration rate estimation in Chinese patients in different stages of chronic kidney disease," Nephrology Dialysis Transplantation, vol. 22, no. 2, pp. 417-423, 2007. 